\title{
Intensive consolidation chemotherapy for acute lymphoblastic leukaemia (UKALL X pilot study)
}

\author{
C R PINKERTON, A BOWMAN, H HOLTZEL, AND J M CHESSELLS
}

Departments of Haematology and Oncology, Pharmacy, and Microbiology, The Hospital for Sick Children, London

SUMMARY Eighty two children with acute lymphoblastic leukaemia presenting at this hospital received one or two modules of intensive chemotherapy to consolidate remission. Modules were given after four and roughly 19 weeks on treatment. Each included two doses of daunorubicin (45 $\mathrm{mg} / \mathrm{m}^{2} /$ day $)$, cytosine arabinoside $\left(100 \mathrm{mg} / \mathrm{m}^{2}\right.$ twice daily $\left.\times 5\right)$, etoposide $\left(100 \mathrm{mg} / \mathrm{m}^{2} /\right.$ day $\left.\times 5\right)$, and 6-thioguanine $\left(80 \mathrm{mg} / \mathrm{m}^{2} /\right.$ day $\left.\times 5\right)$. A total of 132 courses were given. This study included all new patients except girls aged 1-14 years with presenting leucocyte count $<20 \times 10^{9} / 1$. Twenty patients with recurrent disease were also included.

The first 32 patients were given cytosine as a 24 hour infusion, but combined with the other agents this was associated with severe intestinal toxicity, which necessitated a change to a less toxic 12 hourly bolus regimen. The complications of the module are reviewed in terms of myelosuppression, enterotoxicity, infection, and other clinical problems encountered. All patients became profoundly neutropenic and thrombocytopenic. The latter was significantly more severe after cytosine infusion. Overall, 64\% received platelet transfusions and $85 \%$ were re-admitted with fevers requiring intravenous antibiotics for between four and 56 days. Gastrointestinal toxicity with the modified module occurred in $38 \%$ of patients and was severe in $13 \%$.

This intensification module has been adopted by the Medical Research Council Working Party on Childhood Leukaemia for use in a multicentre study (UKALL X) and the details of the problems encountered in the pilot study may be of value to other centres now using this protocol.

Over $90 \%$ of children with acute lymphoblastic leukaemia will enter remission after treatment with vincristine, prednisolone, and asparaginase, ${ }^{1}$ but a large number will subsequently relapse. In 'good risk' girls with common acute lymphoblastic leukaemia and low presenting white blood cell count (WBC), long term survival of around $80 \%$ may be achieved.

For others, however, the outlook is less optimistic, and for boys with a high presenting WBC, long term survival is generally less than $40 \%$

As in other childhood cancer the increased long term survival has accentuated the awareness of the long term side effects of chemoradiotherapy. ${ }^{2}$ Clearer definition of prognostic factors using clinical, immunological, and cytogenetic characteristics may allow the selection of patients for whom less intensive treatment, with fewer long term side effects, is appropriate. The problem of how to improve survival in the intermediate and bad risk patients remains.

In the past few years a number of centres have studied the effect of additional agents given during the induction period. ${ }^{3}$ Few have shown any clear benefit, ${ }^{45}$ but many such regimens have been insufficiently intensive. Although not assessed prospectively, however, it seems likely that the multiagent intensification by the BFM group has improved outcome. ${ }^{6}$

In the current Medical Research Council national trial (UKALL X) the possible advantages of similarly intensive consolidation therapy are being assessed in a randomised manner. A pilot study of a five day module, which comprised daunorubicin, cytosine arabinoside, 6-thioguanine, and etoposide (VP16-213)-'DATE'-incorporated after induction therapy, was started at this hospital in 1983 Here we report a prospective study of toxicity and 
supportive care after this module during the first 18 months of its use.

\section{Patients}

Between March 1983 and July 1984, 72 new cases of acute lymphoblastic leukaemia presented at this hospital. Ages ranged from 1 month to 15 years, and 43 were girls. Thirteen had presenting white blood cell counts $(\mathrm{WBC})>100 \times 10^{9} / 1$ (six T cell, four null, and three common acute lymphoblastic leukaemia). With the exception of 23 'good risk' girls-who had WBC $<20 \times 10^{9} / 1$, were aged $1-14$ years, and were common acute lymphoblastic leukaemia antigen positive-who received standard induction and maintenance therapy, all patients were given either one or two intensive consolidation modules. Four patients with stage III mediastinal T cell lymphoma and 20 with relapsed acute lymphoblastic leukaemia were also included in the study. Nine patients who started treatment before the start of the study were given the late module only (Table 1 ). In total 82 patients received 132 modules up to October 1984.

\section{Methods}

Details of the UKALL $X$ pilot induction regimen are shown in Figure 1 and the consolidation module DATE in Figure 2. The first 25 patients were given cytosine as a continuous infusion over the five days, and all subsequent patients were given 12 hourly intravenous bolus injections.

The elective times for early and late consolidation were at four and 19 weeks, respectively (Fig. 1). The late module was given after two months on maintenance chemotherapy, after cranial irradiation $(18 \mathrm{~Gy})$ - that is, roughly 15 weeks after the early module.

Seven patients were given the early module only.
Table 1 Diagnoses of patients receiving DATE intensification and the respective numbers that were given single modules (early or late) or both early and late

\begin{tabular}{llcccc}
\hline Diagnosis & $(n=)$ & \multicolumn{2}{c}{ Module training } \\
\cline { 3 - 5 } & & & Early & Late & Both \\
\hline $\begin{array}{l}\text { Acute lymphoblastic } \\
\text { leukaemia }\end{array}$ & First remission & $(58)$ & 7 & 9 & 42 \\
T cell lymphoma & Relapsed & $(20)$ & 11 & - & 9 \\
\hline
\end{tabular}

DATE comprised daunorubicin, cytosine arabinoside, 6-thioguanine. and etoposide.

These comprised two children with presenting WBC $100 \times 10^{9} / 1$ who proceeded to bone marrow transplantation, one who died after the early module, two in whom the late module was cancelled due to the morbidity associated with the early module (both low presenting WBC), one girl with a borderline WBC $\left(22 \times 10^{9} / 1\right)$, and one foreign patient in whom the late module was electively omitted.

The first module was given early, at three weeks, in one child with recurrent disease who was slow to achieve remission. The late module was given early in eight patients: four infants under 2 years who did not receive cranial irradiation and in whom irradiation was not repeated and the last module was given 8-12 weeks after the first, and one boy with a high presenting WBC who was not in complete remission at week 4 and was given the late module as soon as the blood count had recovered from the early module.

The early module was delayed in nine patients for the reasons listed in Table 2 . The delay ranged from 1 to 8 weeks and in most cases was due to complications related to treatment during induction.

In 17 patients the late module was delayed more than 16 weeks after the first. In five this was due to

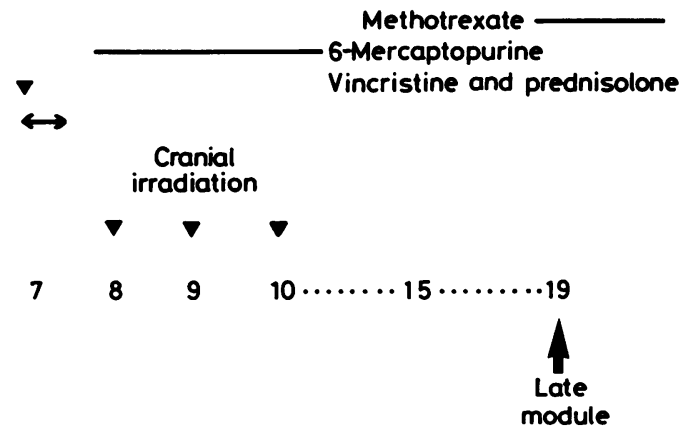

Fig. 1 Details of the drugs given and their timing, during induction, radiotherapy, and early maintenance in UKALL $X$ pilot protocol. 


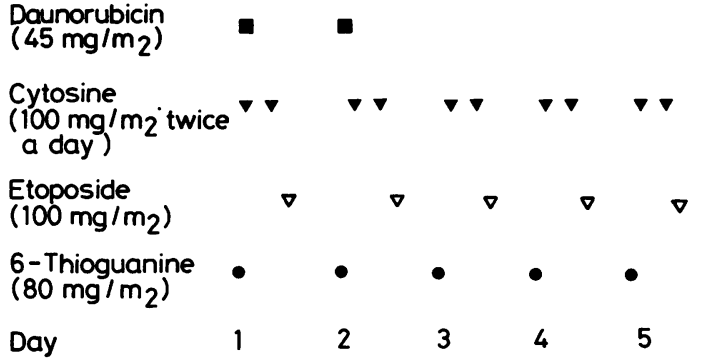

Fig. 2 Details of the constituent drugs in the DATE intensification regimen. With the exception of 6-thioguanine (oral) all were given intravenously (see text for details).

late cranial irradiation as a result of complications associated with the early module; in one irradiation was delayed for a short period until the child was 2 years old and then followed by the late module; in eight the delay was due to severe neutropenia related to intolerance of maintenance therapy started at the time of cranial irradiation; in one the delay was due to herpes zoster infection; and in two patients (both with low presenting WBC) the late module was delayed for one to two weeks for family reasons.

In all cases the blood count had recovered from previous treatment at the time of the module, with neutrophil count $>1 \times 10^{9} / 1$, platelets $>100 \times 10^{9} / 1$, and haemoglobin $>8 \mathrm{~g} / \mathrm{dl}$. The patient's general condition, particularly in relation to nutrition and infection, was taken into account in deciding about the module timing and was balanced against prognostic factors such as age, sex, presenting WBC, and extramedullary disease at diagnosis.

\section{Follow up and management of patients.}

\section{Blood counts and blood product support}

Weekly blood counts were increased to daily once the platelet count had fallen to below $50 \times 10^{9} / 1$, and platelet transfusions were given at below $20 \times 10^{9} / 1$ or if there was appreciable bleeding irrespective of count (four to five packs of platelets $/ \mathrm{m}^{2}$ daily was usually adequate). In ill or febrile patients platelets were given more readily (below $30 \times 10^{9} / 1$ ) and more often because of their reduced effectiveness in such circumstances. Packed red blood cells were given if the haemaglobin concentration fell to below $8 \mathrm{~g} / \mathrm{dl}$. Granulocyte transfusions were considered only in patients with severe localised infection, such as cellulitis or abscess, or bacteriologically proven septicaemia not responding to appropriate treatment with antibiotics.

\section{Management of infections}

In view of the profound myelosuppression a high incidence of infectious complications were anticipated and at the start of the study all patients remained in hospital until the neutrophil count began to recover. Subsequently, all were allowed home after completion of chemotherapy, except infants in whom the signs of infection may be subtle, or where there was any doubt about parental ability to appreciate the need for early re-admission. Parents were advised to take the child's temperature if she or he appeared warm or in any way unwell. A temperature above $38^{\circ} \mathrm{C}$ was regarded as an indication to contact the hospital and usually led to re-admission. Oral decontamination of the bowel was not attempted, nor was protective isolation used.

The investigations carried out in the febrile neutropenic patients were as previously described,${ }^{7}$ with the addition of blood cultures in Sabauroud's medium, serial fungal serology, and viral culture and serology as clinically indicated. Stool samples were routinely screened twice weekly for conventional intestinal pathogens, and the identity and antibiogram of the aerobic Gram negative bowel flora were also determined. The results of the latter investigations were used to determine whether initial treatment with antibiotics should be the first or second line antibiotic policy of the unit. In the

Table 2 Complications during induction chemotherapy in nine patients that led to delay in administration of early intensification. The treatment given during the interim period is listed

\begin{tabular}{llll}
\hline $\begin{array}{l}\text { Case } \\
\text { No }\end{array}$ & $\begin{array}{l}\text { Weeks } \\
\text { delay }\end{array}$ & Reason & Interim treatment \\
\hline 1 & 1 & Perforated appendix & Vincristine, prednisone \\
2 & 1 & Veg abscess & Vincristine, prednisone \\
3 & 1 & Septicaemia and diarrhoea & Vincristine, prednisone \\
4 & $1 \cdot 5$ & Prolonged myelosuppression & Vincristine, prednisone \\
5 & 1 & Diarrhoea, vomiting, and weight loss & Started maintenance \\
6 & 8 & Wound infection and weight loss & Started maintenance \\
7 & 5 & Acute epiglotitis and weight loss & Started maintenance \\
8 & 4 & Encephalopathy and weight loss & Started maintenance \\
9 & 8 & Septicaemia and malnourished & \\
\hline
\end{tabular}


absence of piperacillin resistant organisms piperacillin $300 \mathrm{mg} / \mathrm{kg} /$ day and gentamicin $8 \mathrm{mg} / \mathrm{kg} /$ day were used. When indicated by the antibiogram ceftazidime $50 \mathrm{mg} / \mathrm{kg} /$ day was substituted for piperacillin. Intravenous metronidazole was given to patients with severe diarrhoea. Oral nystatin was routinely prescribed.

If the temperature settled promptly and no causative agent was found treatment with antibiotics was stopped when the child had been afebrile for 48 hours, irrespective of the blood count. Documented bacteraemia was followed by a 10 day course of appropriate treatment with antibiotics.

In patients in whom fever failed to settle within five days of treatment with antibiotics, appropriate for cover of the aerobic Gram negative bowel flora, intravenous amphotericin was used. Early treatment was influenced by the presence of candida in gut, mouth, or skin and was reviewed in the light of clinical response and serial candida serology estimated every three to five days. The duration of treatment with amphotericin was based on the documentation of presence or absence of systemic candida infection, which was indicated by a rise in antigen or antibody, or both. Where systemic sepsis was documented a course of six weeks' treatment was given. Where three serial estimations of antigen and antibody failed to reveal evidence of systemic disease treatment was stopped after two to three weeks or on recovery of the neutrophil count.

\section{Enterotoxicity}

As with suspected infection a low threshold for re-admission was maintained and parents were advised to contact the hospital in the event of any intestinal symptoms. If diarrhoea developed the child was re-admitted for observation and early intravenous fluids given if symptoms did not settle. With severe enterotoxicity, parenteral nutrition was begun early, particularly in patients whose nutritional state was already suboptimal.

\section{Results}

As the amount of previous treatment might have influenced toxicity patients have been subdivided with regard to the timing of the module-that is, late module only, early module, or second module given to the same patient (repeat late module). The method of administration of cytosine is also considered and whether the patient was in first or subsequent remission. The times given refer to the number of days after starting the module. Statistical differences between groups were assessed using the $\chi^{2}$ test.

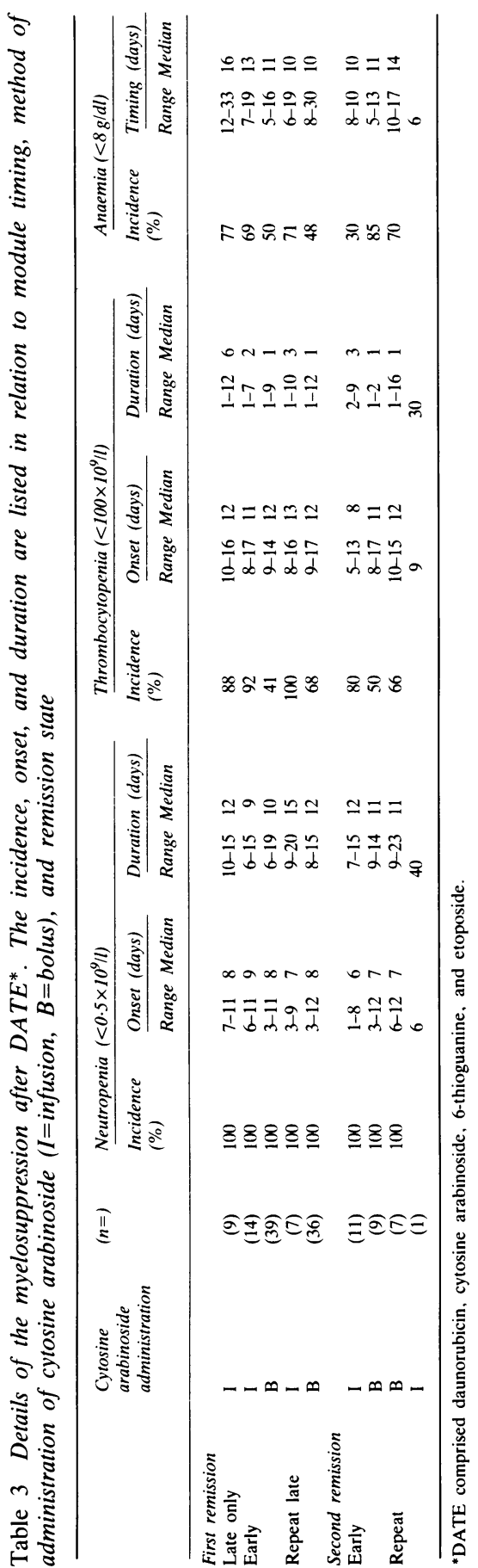


Myelosuppresion. Profound myelosuppression was an invariable feature after treatment (Table 3). The neutrophil count fell to below $0.5 \times 10^{9} / 1$ in all patients and to undetectable numbers in most. The onset of neutropenia ranged from day 7-12 in new cases and 1-12 in patients with recurrent disease and the duration of neutropenia from 6-20 and 7-40 days, respectively. Severe thrombocytopenia occurred in many patients, the timing of onset ranging from day 5-17. Sixty four per cent of patients required platelet transfusions, and platelet requirements were higher in cases who had received cytosine as an infusion rather than as a bolus $(88 \% v$ $56 \%, \mathrm{p}<0.001)$. Bruising and petechiae were not uncommon, but with the exception of intestinal haemorrhage in patients with severe enterotoxicity there were no serious bleeding problems. In three patients myelosuppression was particularly severe, lasting over three weeks. Two of these had recurrent disease and had been heavily pretreated. The other, a new case, developed severe enterotoxicity and septicaemia and, despite initial partial recovery of blood count, remained profoundly neutropenic and thrombocytopenic and developed candida septicaemia. After eight weeks his count had completely recovered and he proceeded into maintenance therapy.

Enterotoxicity. This was graded according to severity (Table 4). The timing of onset of symptoms ranged from day 5-16 and their duration from 1-35 days (Table 4).

Characteristically, patients remained well during the administration of the module apart from some nausea and vomiting associated with daunorubicin. Cytosine was in general well tolerated with no conjunctival, skin, or neurological reactions.

The commonest intestinal symptom was abdom- inal pain, beginning a few days after completing treatment, although in some patients anorexia and vomiting were prominent. Pain was poorly localised, with few local signs. Diarrhoea ranged from a couple of loose stools, settling after a day or two, to watery diarrhoea, passed several times a day associated with intermittent crampy abdominal pain. In severe cases, the stool was mucous or blood stained and complete casts of intestinal mucosal lining were occasionally passed in the stool. Abdominal distension was a common feature and was usually of gaseous origin compounded by some degree of ileus. Plain $x$ ray film showed distended loops of small bowel, and in one case intramural gas was apparent. In severe cases ascites contributed to the distension, in part as a consequence of intestinal protein loss. Oral mucosal ulceration was rarely seen. When cytosine was given as a continuous infusion over five days $29 \%$ of patients developed severe grade III or IV enterotoxicity, and this contributed largely to the death of one child. The administration of cytosine was therefore modified to a daily injection and this reduced the incidence of grade III or IV toxicity to $13 \%(p<0 \cdot 001)$. Moreover, with the bolus regimen, $60 \%$ had no intestinal symptoms compared with $24 \%$ after the infusion $(p<0 \cdot 001)$.

Infection. Modules were followed by fever with neutropenia that required intravenous antibiotics in $85 \%$ of cases. The range of organisms isolated in blood cultures was similar to that previously reported from this unit, ${ }^{7}$ with Escherichia coli, Pseudomonas, and Staphylococcus epidermidis or Staph. aureus predominating. The duration of treatment with antibiotics ranged from four to 25 days (median nine days). Several patients developed localised staphylococcal cellulitis, usually at an intravenous access site. One such patient, in whom

Table 4 Onset, duration, and severity of enterotoxicity after DATE

\begin{tabular}{|c|c|c|c|c|c|c|c|c|c|c|}
\hline & \multirow{2}{*}{$\begin{array}{l}\text { Cytosine } \\
\text { arabinoside } \\
\text { administration }\end{array}$} & \multirow[t]{2}{*}{$(n=)$} & \multicolumn{2}{|c|}{ Onset (days) } & \multicolumn{2}{|c|}{ Duration (days) } & \multicolumn{3}{|c|}{ Toxicity grading } & \multirow{2}{*}{$\begin{array}{l}\text { Requiring intravenous } \\
\text { feeding }\end{array}$} \\
\hline & & & Range & Median & Range & Median & 0 & $1 / 11$ & $111 / 1 \mathrm{~V}$ & \\
\hline \multicolumn{11}{|c|}{ First remission } \\
\hline Late only & I & (9) & $6-16$ & 11 & $2-10$ & 3 & 1 & 6 & 2 & 1 \\
\hline \multirow[t]{2}{*}{ Early } & I & (14) & $8-14$ & 13 & $1-9$ & 3 & 6 & 5 & 3 & 1 \\
\hline & B & $(39)$ & $3-9$ & 6 & $2-15$ & 7 & 21 & 8 & 8 & 4 \\
\hline \multirow[t]{2}{*}{ Repeat late } & I & (7) & $5-13$ & 9 & $2-14$ & 6 & 0 & 4 & 3 & 1 \\
\hline & B & (36) & $6-14$ & 8 & $2-35$ & 3 & 24 & 8 & 3 & 2 \\
\hline \multicolumn{11}{|c|}{ Second remission } \\
\hline \multirow[t]{2}{*}{ Early } & I & (11) & $5-16$ & 10 & $2-15$ & 4 & 4 & 4 & 3 & 3 \\
\hline & B & (9) & $8-9$ & 8 & $3-4$ & 3 & 6 & 3 & 0 & 0 \\
\hline \multirow[t]{2}{*}{ Repeat } & B & (7) & $9-10$ & 9 & $9-10$ & 9 & 4 & 3 & 0 & 0 \\
\hline & I & (1) & 10 & & 35 & & & & 1 & 1 \\
\hline
\end{tabular}

Intestinal toxicity grading: 1 Occasional crampy pain; 11 Cramps and two to three loose motions each day, oral replacement; 111 Severe diarrhoea requiring intravenous fluids; 1V Florid enterocolitis, usually requiring parentral feeding.

Cytosine arabinoside administration: $\mathrm{I}=$ infusion, $\mathrm{B}=$ bolus. DATE comprised daunorubicin, cytosine arabinoside, 6-thioguanine, and etoposide. 
blood cultures yielded positive results, received granulocyte transfusions. Six patients, four of whom had been given cytosine by infusion, received amphotericin because of unremittent fever, but in one case this was for severe candida oesophagitis. The duration of antifungal treatment ranged from five to 56 days (median 14 days). One fifth of the modules were followed by the use of intravenous metronidazole, mostly in the earlier patients in whom intestinal toxicity was more severe. Acyclovir was given in seven cases: in two as empirical treatment in unexplained encephalopathy but without firm supportive evidence for herpes infection, in one for severe facial herpes zoster, and in four for herpes simplex stomatitis.

Mortality. Two patients died after the unmodified module (infusion of cytosine) and one after the modified (bolus cytosine), an overall mortality of $3.6 \%$. Both of the patients who died after the unmodified module had recurrent disease.

One girl, aged 13 years, with isolated central nervous system relapse, developed a fever on day 9 , which persisted in hospital despite treatment with antibiotics. She died on day 11 with a fulminant $E$. coli septicaemia and intramesenteric haemorrhage.

A 4 year old girl, who had relapsed in the bone marrow on maintenance therapy, collapsed and died within a few hours of admission to her local hospital with a fever and mild diarrhoea that developed 48 hours after completing treatment. Although blood cultures yielded negative results, it was assumed this death was due to septicaemia. Postmortem examination revealed no specific cause and in particular no evidence of cardiomyopathy (she had received 315 $\mathrm{mg} / \mathrm{m}^{2}$ daunorubicin).

The child who died in first remission was a 2 year old girl with a presenting WBC of $231 \times 10^{9} / 1$. She had a very stormy induction period, developing septicaemia and enterotoxicity, the latter probably due to the initial treatment with daunorubicin. Because of these problems the early module was delayed until week 5. Despite this, she developed diarrhoea on day three of the module and died on day 15 with fulminant Pseudomonas aeruginosa septicaemia.

\section{Discussion}

As might be expected with any intensive combination chemotherapy regimen the morbidity associated with the DATE module was high. With the modified form, $85 \%$ of patients required readmission to hospital because of fever, and $13 \%$ developed severe enterotoxicity. In some patients abdominal pain was a major problem and, although moderate discomfort usually responded to simple analgesics or antispasmodics, opiates were required in severe cases, although given with caution where there was florid enterocolitis. In general no attempt was made to control diarrhoea with pharmacological agents. Most are ineffective in cytotoxic drug induced enteritis, although loperamide may have a role. The effect of this drug on gut motility may give rise, however, to misleading clinical signs, as in one child where an apparent improvement in diarrhoea after loperamide was followed by sudden massive loss of pooled fluid and profound hypokalaemia. Secondary clostridial infection should be anticipated and metronidazole or vancomycin may be indicated where there is a bloody diarrhoea. In severe cases the abdominal signs may resemble those of an 'acute surgical abdomen' with guarding and rebound tenderness, but there is rarely any indication for surgical intervention. The exception is clear radiological evidence of perforation where local repair may be possible or severe intestinal haemorrhage where resection of involved gut may be necessary. ${ }^{8}$ Otherwise, laparotomy in these immunosuppressed, thrombocytopenic, and often septic children is likely to reveal an oedematous, haemorrhagic gut only, and the postoperative morbidity is high. Other, unrelated causes of an acute abdomen should also be borne in mind. In this series one child developed acute appendicitis in the week after treatment. Regular review by a paediatric surgeon with experience of oncology patients is necessary in an often delicate balance between timely or unnecessary intervention.

Cytosine has been implicated as a major cause of intestinal complications after combination chemotherapy. ${ }^{9}$ As a single agent myelosuppression is usually the dose limiting factor, ${ }^{10}$ but in combination with daunorubicin severe intestinal problems often arise. ${ }^{11}$ As the DATE module has been designed for intermediate as well as high risk patients it was considered that severe toxicity in about one third of patients was unacceptable. The reduction in toxicity associated with the change from cytosine infusion to bolus is consistent with its short plasma half life. ${ }^{12}$ This allows some recovery of intestinal crypt cells between doses. To what extent this modification alters therapeutic activity remains speculative, although there is some clinical evidence that cytosine infusion is more effective. ${ }^{13}$

Enteral feeding was invariably stopped during the acute phase as at this time there is usually severe villus atrophy. ${ }^{9}$ During the recovery phase, however, energy supplemented, hypoallergenic diets may be useful in regrading to full oral feeds.

Intravenous feeding was usually given using 
peripheral venous access. In a small number of patients central silastic lines were inserted percutaneously. In general central catheters such as Hickmann's catheters were not electively used and with the change from cytosine infusion the need for intravenous feeding diminished markedly.

In the ill patient close monitoring of electrolyte balance was essential as hyponatraemia, hypokalaemia, hypomagnesaemia, uraemia, and a variety of other abnormalities occurred. Metabolic derangements and renal dysfunction were often compounded by the effects of amphotericin and the aminoglycosides. Careful surveillance of blood concentrations of potentially nephrotoxic agents was clearly mandatory.

The early use of combined broad range antibiotics has reduced the mortality in patients with neutropenia. ${ }^{15}$ The myelosuppression after DATE was severe, requiring close bacteriological surveillance and rapid treatment in the event of fever. When the period of neutropenia is particularly prolonged the risk of fungal infection is high and for this reason a low threshold for starting parenteral amphotericin $\mathrm{B}$ (the agent of choice ${ }^{14}$ ) is advisable. ${ }^{15}$

Although it is preferable to give early intensification soon after the blood count has recovered (particularly in patients with a poor prognosis), there may be clinical indications for some delay (Table 2). Such complications are not uncommon with current four drug induction regimens. Nutrition was a particular problem, and active measures should be taken to prevent excessive weight loss in patients who are ill during induction. Supplementary enteral feeds and early intravenous feeding may be needed before intensification treatment. With appropriate supportive care, the mortality of DATE should be low $(\approx 2-3 \%)$, comparable with that for other similar intensification regimens. ${ }^{4}$ The additional stress that such treatment puts on the families involved is clearly an important consideration and appropriate psychosocial support is needed. Only the current randomised Medical Research Council study will answer the question about whether the additional problems encountered by all concerned are justified by an improved prognosis.
We are indebted to the nursing and junior medical staff of the haematology and oncology unit at the Hospital for Sick Children and the many others involved in the supportive care team.

CRP was in receipt of a Clinical Research Fellowship from the Leukaemia Research Fund.

We are grateful to Mrs Reynaud for help with preparation of the manuscript.

\section{References}

${ }^{1}$ Miller DR, Leikin S, Albo V, Sather H, Karon M, Hammond D. Prognostic factors and therapy in acute lymphoblastic leukaemia of childhood. Cancer 1983;51:1041-9.

2 Chessells JM. Childhood acute lymphoblastic leukaemia: the late effects of treatment. Br J Haematol 1983;53:369-78.

${ }^{3}$ Chessells JM. Risks and benefits of intensive treatment of acute leukacmia. Arch Dis Child 1985;60:193-5.

${ }^{4}$ Sackmann-Muriel F, Svarch E, Eppinger-Helft M, et al. Evaluation of intensification and maintenance programmes in the treatment of acute lymphoblastic leukaemia. Cancer 1978;42:1730-40.

5 Camittal BM, Pinkel D, Thatcher LG, Casper J, Kun LE, Lauer S. Failure of early intensive chemotherapy to improve prognosis in childhood acute lymphocytic leukaemia. Med Pediatr Oncol 1980;8:384-9.

${ }^{6}$ Lampert F, Henze G, Langermann HJ, Schellong G, Gadner H, Reihm HJ. Acute lymphoblastic leukaemia: current status of therapy in children. Recent Results Cancer Res 1984:93:159-81.

${ }^{7}$ Chessells JM, Leiper AD. Infection during remission induction in childhood leukaemia. Arch Dis Child 1980;55:118-23.

* Schaller RT Jr, Schaller JF. The acute abdomen in the immunologically compromised child. J Pediatr Surg 1983; 18:937-43.

${ }^{9}$ Slavin RE, Dias MA, Saral R. Cytosine arabinoside induced gastrointestinal toxic alterations in sequential chemotherapeutic protocols. Cancer 1978;42:1747-59.

10 Ochs J, Sinkule JA, Danks MK, Look AT, Bowman WP, Rivera G. Continuous infusion high-dose cytosine arabinoside in refractory childhood leukemia. Journal of Clinical Oncology 1984;2:1092-7.

" Johnson H, Smith TJ, Desforges J. Cytosine-arabinosideinduced colitis and peritonitis: nonoperative management. Journal of Clinical Oncology 1985;3:607-12.

12 Slevin ML, Piall EM, Aheren GW, Johnston A, Lister TA. The pharmacokinetics of cytosine arabinoside in the plasma and cerebrospinal fluid during conventional and high dose therapy. Med Pediatr Oncol 1982;10:(Suppl):157-68.

13 Rai KR, Holland JF, Glidwell OJ, Weinberg V, Brunner K, Obrecht JP. Treatment of acute myeloid leukaemia: a study by cancer and leukaemia group B. Blood 1981;58:1203-12.

14 Cohen J. Antifungal chemotherapy. Lancet 1982;ii:532-7.

15 Pizzo PA, Robichaud KJ, Gill FA, Witebsky FG. Empiric antibiotic and antifungal therapy for cancer patients with prolonged fever and granulopenia. Am J Med 1982;72:101-11.

Correspondence to $\operatorname{Dr}$ C R Pinkerton, Department of Haematology and Oncology, Hospital for Sick Children, Great Ormond Street, London.

Received 12 August 1986 